\title{
REA_15 - Fragment production of the spike protein from SARS-CoV-2 in insect cells for the development of serological diagnosis
}

Natália Salazar de Castro ${ }^{*}$; Thiciany Blener Lopes ${ }^{1}$; Bruno Cassaro ${ }^{1}$; Flávio Guimarães da Fonseca ${ }^{1}$; Ana Paula Salles Moura Fernandes ${ }^{1}$; Santuza Maria Ribeiro Teixeira ${ }^{1}$; Ricardo Tostes Gazzinelli².

${ }^{1}$ UFMG - Universidade Federal de Minas Gerais;

${ }^{2}$ Fiocruz - Fundação Oswaldo Cruz.

Introduction: The world has been devastated by the spread of the new coronavirus. Thousands of lives are lost daily and the economic impact has been incalculable. Diagnosing quickly means one of the point to control the progress of the disease, as monitoring the antibody levels of disease development and vaccinated people. Thus, serological tests such as ELISA and immunochromatographic test are being developed and constantly improved. Producing recombinant antigens capable of being recognized with high sensitivity and specificity by antibodies in different stages of this disease, as infected, curate and vaccinated are the major challenge in the process of developing serological tests. Prokaryotic systems for expression of recombinant proteins are widely used because of their simplicity, short production time and low cost. However, eukaryotic systems are the only choice to conserve the structural features as post-translational modifications and native conformation, as insect and mammalian cells. In this context, the insect cells are simple, low cost, faster with significant expression protein level. The spike protein or fragments applied for Covid-19 diagnosis expressed in the eukaryotic system has been widely used. In Brazil, for most tests developed, this protein has being imported with high costs and long delivery times, which reinforces the need for national production.

Objective: Express a fragment of the Spike protein from SARS-CoV-2 in insect cells transfected with recombinant baculovirus and evaluate its potential as an antigen for the development of serological diagnostic tests.

Methodology: The Bac-to-BacR HBM TOPO Secreted Expression System (Invitrogen) was chosen to construct the system. Briefly a gene segment encoding the sequence of interest for the Spike protein wascloned into the pFastBac/HBM-TOPO plasmid (Invitrogen). Competent DH10Bac E. coli cells containing the baculovirus genome were transformed with plasmid construction for generating the recombinant viral genome. Then cultured Sf9 insect cells were transfected with recombinant bacmid purified for the generation of the recombinant vírus and expression of the protein of interest, expressed in fusion with six histidines for purification by affinity chromatography and with a signal peptide, for secretion in the culture médium. Purified protein samples were analyzed by $12,5 \%$ SDS-PAGE and Western Blot. After purification, the protein was evaluated by ELISA for their ability to be recognized by antibodies in the serum of individuals infected with Sars-coV-2.

Results: The protein was expressed in the infected cells as the recombinant virus and purified from the culture supernatant. In ELISA assays for IgG evaluation the preliminary results fixing $500 \mathrm{ng}$ of protein per well and analyzing 16 positive and 16 negative samples showed $100 \%$ specificity and $73 \%$ sensitivity.

Conclusion: The initial results led us to intensively explore the potential of the insect cell/baculovirus expression system for large-scale production of SARS-CoV-2 antigens for the development of national serological tests.

Keywords: Coronavirus; Spike; Recombinant 